Accepted manuscript - Optometry \& Vision Science $22^{\text {nd }}$ May 2019

2

3

4

\title{
Increased word spacing improves performance for reading scrolling text with central vision loss
}

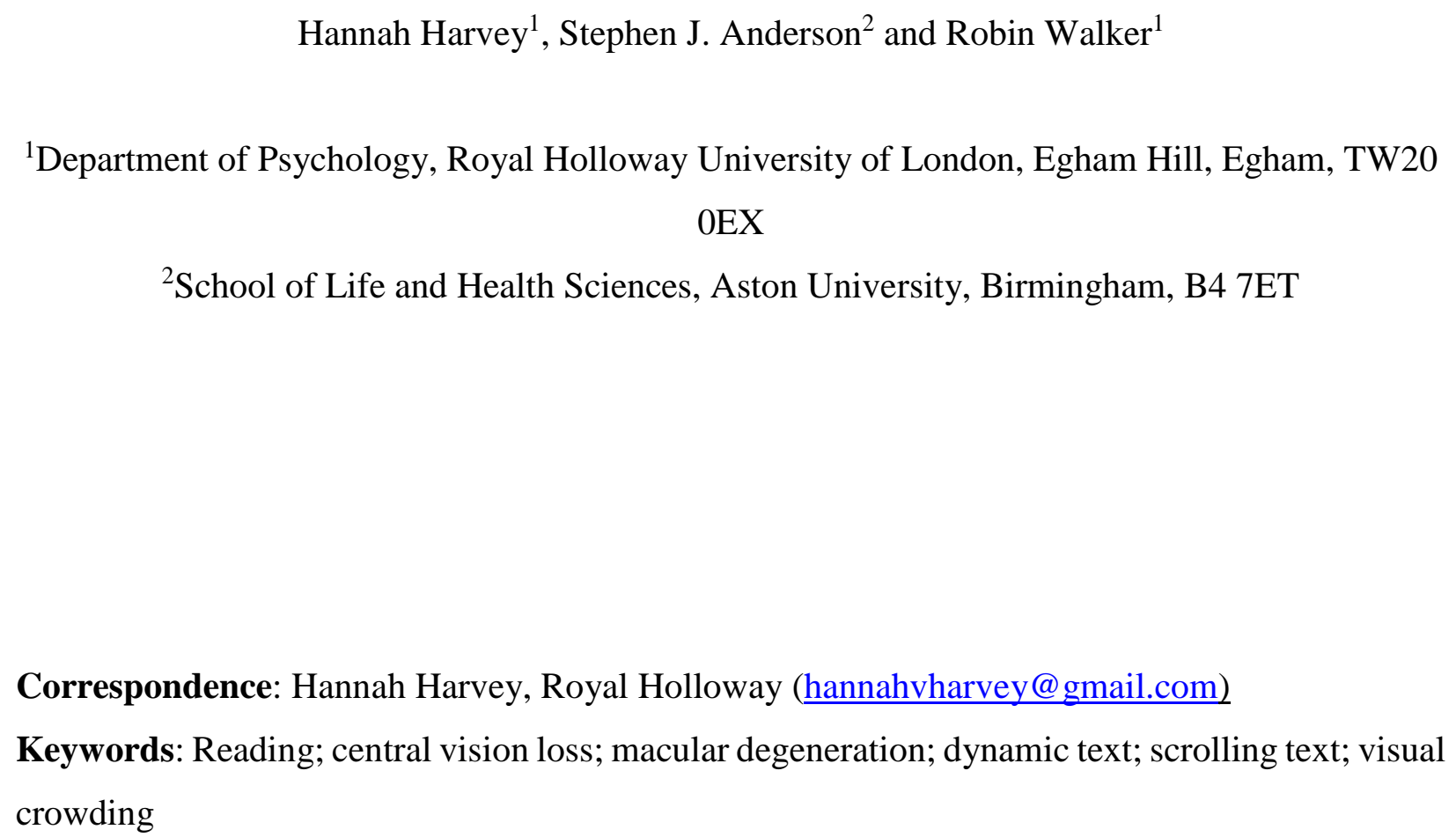
crowding

Disclosure: The authors report no conflicts of interest and have no proprietary interest in any of the materials mentioned in this article.

Acknowledgements: This work was supported by a Royal Holloway Reid studentship to Hannah Harvey. 


\section{Visual crowding and scrolling text} 37 rehabilitation practitioners.

\section{Abstract} three characters. rate (in wpm). loss.

Significance: Scrolling text can be an effective reading aid for those with central vision loss. Our results suggest that increased inter-word spacing with scrolling text may further improve the reading experience of this population. This conclusion may be of particular interest to low vision aid developers and visual

Purpose: The dynamic, horizontally scrolling text format has been shown to improve reading performance in individuals with central visual loss. Here, we sought to determine whether reading performance with scrolling text can be further improved by modulating inter-word spacing to reduce the effects of visual crowding: a factor known to impact negatively on reading with peripheral vision.

Methods: The effects of inter-word spacing on reading performance (accuracy, memory recall and speed) was assessed for eccentrically-viewed single sentences of scrolling text. Separate experiments were used to determine whether performance measures were affected by any confound between inter-word spacing and text presentation rate in words per minute (wpm). Normally-sighted participants were employed, with a central vision loss implemented using a gaze-contingent scotoma of $8^{\circ}$ diameter. In both experiments, participants read sentences that were presented with an inter-word spacing of one, two or

Results: Reading accuracy and memory recall were significantly enhanced with triple-character inter-word word spacing (both measures $P \leq 0.01$ ). These basic findings were independent of the text presentation

Conclusions: We attribute the improvements in reading performance with increased inter-word spacing to a reduction in the deleterious effects of visual crowding. We conclude that increased inter-word spacing may enhance reading experience and ability when using horizontally scrolling text with a central vision 


\section{Visual crowding and scrolling text}

\section{Introduction}

Horizontally scrolling text has been shown to be a useful technique for reducing the level of reading difficulty and discomfort typically experienced by individuals with central vision loss. ${ }^{1-5}$ Most commonly seen in rolling news tickers, this text format can be applied as a reading aid for individuals with macular dysfunction, either manually using CCTV (Closed-Circuit Television) aids ${ }^{6}$ or via mobile apps such as the MD_evReader. ${ }^{2}$ There are several reasons why this is the case. First, scrolling text allows readers to limit active oculomotor navigation of the text, a factor known to be a significant challenge for people without central vision. ${ }^{7}$ Second, because scrolling text is presented as a single line, it not only removes the difficulty associated with navigation of multi-lined text with a central scotoma, ${ }^{8}$ but also negates the deleterious effects of inter-line crowding. ${ }^{9}$ Crowding refers here to the phenomenon in which identification of a target word is significantly impaired by the presence of nearby words. ${ }^{10-12}$ Third, visual acuity for dynamic stimuli may be superior to that for static stimuli at some eccentricities when presented at a reasonable rate. ${ }^{13}$ Finally, scrolling text may allow individuals without central vision to reduce their fixational instability ${ }^{14}$ by holding fixation in an eccentric location so that the text can move through an optimal part of their remaining visual field (i.e. their preferred retinal locus [PRL]); this is similar to a viewing technique ('steady-eye strategy') which has been advocated by some low-vision practitioners to improve reading performance. ${ }^{15}$

Although reading a single line of drifting text cannot involve the influence of inter-line crowding, it may be adversely affected by inter-word crowding. The latter may impact significantly on reading performance with peripheral vision. Sufficient word spacing is naturally required for the delineation of word boundaries; ${ }^{16}$ increased overall reading times and increased difficulty with word identification arise when typical word spacing information is removed. ${ }^{17}$ However, due to the negative effects of visual crowding, ${ }^{18-20}$ which are known to worsen as retinal eccentricity increases, ${ }^{21}$ standard inter-word spacing 
80 (of a single-character space) may be insufficient to allow identification of individual words within a 81 passage of eccentrically-viewed text. crowding for reading with central vision loss, reporting that increased word and line spacing within multi-

84 line passages of static text improves reading performance in individuals with macular disease. They assessed reading speed with single, double or triple word/line spacing, and observed the fastest reading speeds for text with double line and double word spacing. The effects of horizontal word crowding on

87 reading performance with scrolling text is not known.

91 attentional deployment during periods of pursuit have shown that the effects of crowding may be

92 increased for stimuli positioned behind the direction of pursuit. ${ }^{25}$ This is broadly comparable to the

93 situation for upcoming words with scrolling text (see Figure 1), suggesting that word crowding in 94 peripheral vision may be more problematic with scrolling text than static text. If this is the case, reading performance with horizontally scrolling text may be enhanced by increasing inter-word spacing.

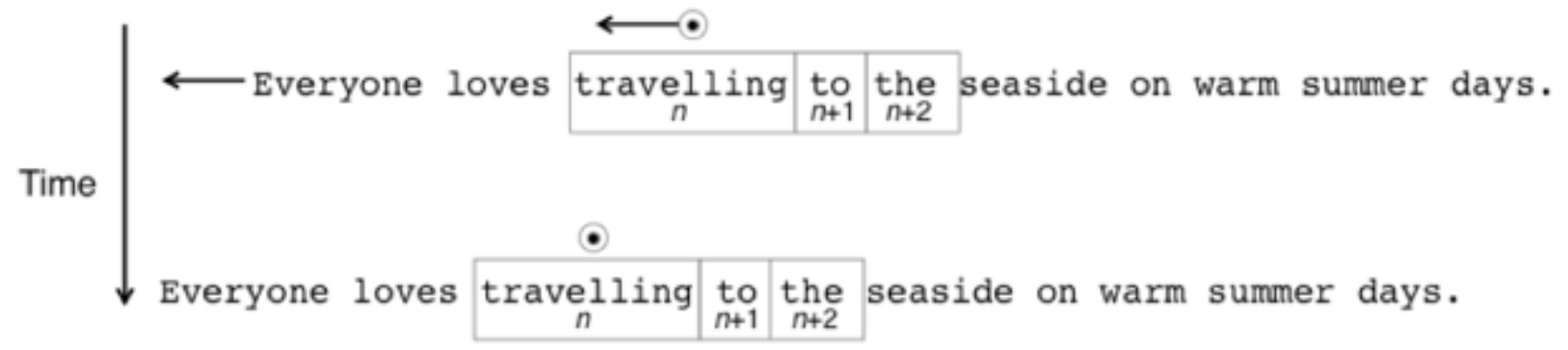

Figure 1. Processing of upcoming words in the parafoveal area (e.g. word $n+1$ and word $n+2)$ may be

97 disrupted by increased crowding of text positioned behind the direction of movement. 


\section{Visual crowding and scrolling text}

The aim of the present study was to investigate the effects of inter-word spacing on reading

performance for scrolling text under conditions of central vision loss. The latter was achieved using a gazecontingent central scotoma of $8^{\circ}$ diameter. Our study followed the general approach of Blackmore-Wright et al. $^{9}$ in that we compared single-, double- and triple-character inter-word spacing when reading eccentrically-viewed text. The measures of visual performance included reading accuracy (i.e. reading error rate), sentence recall, and reading speed (in words per minute, wpm).

\section{Methods}

Twelve students were recruited for each experiment (Expt. 1: 8 females, group mean age 19.5 years; Expt. 2: 11 females; group mean age 24.1 years). No participants took part in both studies. A-priori power calculations based on the effect size for inter-word spacing recorded by Blackmore-Wright et al. ${ }^{9}$ were performed using $\mathrm{G}^{*}$ Power software ${ }^{26}$ indicating that this sample size should provide adequate statistical power to detect this effect of interest. All participants were native English speakers from Royal Holloway, University of London, with no reading or language impairments. All had self-reported normal or corrected-to-normal vision, received course credit for their participation, and all gave prior informed consent as approved by departmental ethical review at Royal Holloway. This study adhered to the tenets of the Declaration of Helsinki.

Participants were required to read text under conditions of a simulated central vision loss, which was imposed as a gaze-contingent circular scotoma of $8^{\circ}$ diameter, as described below. The main manipulation of interest was inter-word spacing, set to one, two or three characters. Two experiments were employed to assess the effects on reading performance of any potential confound between text drift rate and inter-word spacing. 
In both experiments, text was presented as a single scrolling line that moved smoothly across the

123 screen from right to left, at a fixed speed on each trial. The fixed speeds were set differently in Experiments

1241 and 2, constituting the only major difference between these studies. In Experiment 1, text was scrolled

125 in every trial at a fixed speed of two pixels per screen refresh $(6.7 \%$ s; which equates to approximately 9.1

126 characters/s). The increased inter-word spacing necessarily reduced text scrolling speed, with single,

127 double and triple spacing conditions yielding display speeds of approximately 109, 91 and 78 wpm,

128 respectively. In order to ensure that the slowing display speed with increased word spacing was not a

129 confound in our results, the text in Experiment 2 was scrolled at a speed of approximately 91 wpm for all

130 three spacing conditions, with the pixel-scrolling rate modified across conditions to compensate for the

131 delayed rate of presentation produced by wider inter-word spacing. Single-spaced text was therefore

132 scrolled at $3.8 \%$, double-spaced text at $4.6 \%$, and triple-spaced text at $5.1 \%$ s.

133 Stimuli were displayed on a computer monitor (refresh rate $100 \mathrm{~Hz}$ ) as black text (24pt Courier

134 font) on a white background. The Courier font has been identified as suitable for reading with central

135 vision loss, ${ }^{27,28}$ and is a fixed-width font with each character (including inter-word spaces) of the same

136 horizontal extent. The character extent ( $x$-height) in this study was $0.6^{\circ}$, four times the letter acuity

137 threshold at $4^{\circ}$ eccentricity, ${ }^{29}$ and larger than the expected critical print size (CPS), assuming a CPS acuity

138 ratio of $2: 1 .^{30}$ Viewing distance was maintained at $70 \mathrm{~cm}$ using a table-mounted head restraint, which also

139 served to stabilise head position. Note that a chinrest was not used as jaw movements made with

140 vocalisations could potentially disrupt eye-tracking measures. Participants were advised to adopt a

141 vertical PRL as this improves performance when reading horizontally scrolling with a central vision loss. ${ }^{3}$

142 A horizontal guide-line positioned $4^{\circ}$ above the text was used to aid adherence to the advised PRL (see

143 Figure 2). 


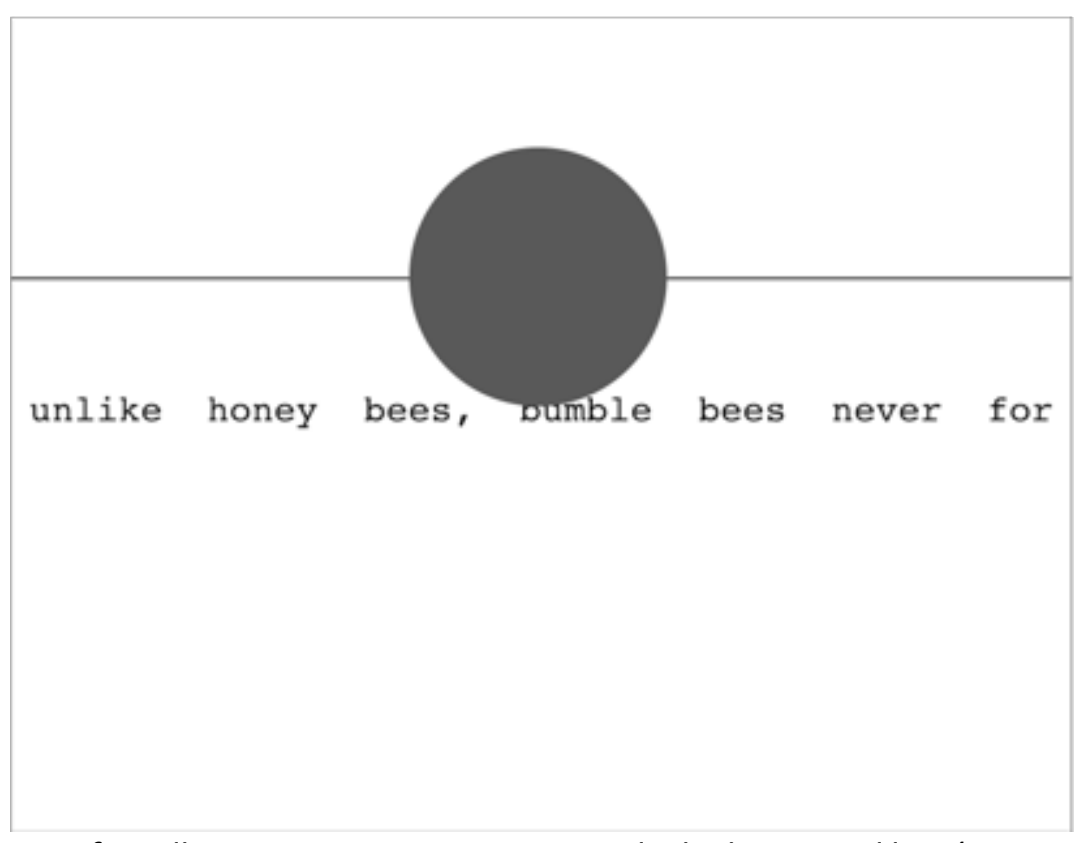

Figure 2. Schematic of scrolling text presentation protocol. The horizontal line (positioned $4^{\circ}$ above the top of the text display area) was used to encourage participants to adhere to the eccentric viewing strategy (upper vertical PRL).

A set of 290 similarly constructed sentences of average length 11.0 words (SD 1.2) was employed across both experiments. All sentences were based on the MNRead corpus. ${ }^{31}$ The average number of characters in each word was 5.3 (SD 0.6). Sentences were randomly allocated into blocks which were allocated to each of the inter-word spacing conditions, with this allocation counterbalanced across participants so that all sentences appeared equally in each spacing condition. Inter-word spacing was set uniformly across a sentence as one, two or three character spaces.

During reading, monocular (right-eye) eye movements were recorded using an EyeLink 1000 video-based eye tracker at a sample rate of $1000 \mathrm{~Hz}$. Except when a blink was detected, eye position was used to re-draw a scotoma every $10 \mathrm{~ms}$ based on the last sample location. If a blink was detected, the scotoma was redrawn continuously in the same position until the blink ended. The scotoma was developed and displayed as a homogenously filled grey circle following recommendations made by Aguilar and Castet $^{32}$ to address issues of pupil size changes (e.g. due to blinks) that are detrimental for gaze- 
161 contingent scotoma paradigms. Prior to each trial, a standard Eyelink drift-checking procedure was

162 performed in the absence of the gaze-contingent scotoma using a gaze-fixation target positioned $4^{\circ}$ above

163 the location where the text would subsequently appear. This required the participant to adopt the correct

164 eccentric fixation location prior to reading. Following this, as an additional means of ensuring gaze position

165 accuracy, a gaze-contingent landmark ( $0.8^{\circ}$ black square) was presented in the same spatial location as

166 the drift-checking target, requiring stable fixation in this region for at least $40 \mathrm{~ms}$ before the trial would

167 begin. The importance of minimising head movements was stressed to participants before and during 168 each experimental block of trials. On rare occasion $(<5 \%$ of trials), however, recalibration was required 169 as one or the other verification stages indicated a loss of position accuracy.

171 to present the stimuli. Prior to an experimental block, a practice block was completed to allow participants

172 to (re-) familiarise themselves with the eccentric reading task - the practice block was drawn from a pool

173 of unused sentences. Presentation of each spacing condition was randomised for each participant.

174 Participants were asked to read aloud each presented sentence, and recall the sentences aloud at the 175 conclusion of each trial. An auditory recording of the session was made for later scoring. Reading accuracy 176 was determined from the number of errors made while reading each sentence. Errors were identified as 177 omissions (e.g. She could not sleep in the same room as the big [scary] clown), substitutions (e.g. We like 178 feeding carrots to the rabbits [horses] that live in that field) or insertions (e.g. My sister was going to play 179 [by] the piano but it was broken). This procedure allowed measures of reading speed, accuracy and 180 memory to be analysed (using $\mathrm{R} 3.4 .4^{33}$ ). Statistical analysis of the effects of inter-word spacing was 181 completed using a within-subjects one-way ANOVA. Multiple comparisons were corrected using 182 Bonferroni's method, and effect sizes are reported as generalised eta squared $\left(\eta_{\mathrm{G}}{ }^{2}\right)$ or Cohen's $d$ where 183 appropriate.

\section{Results}




\section{Visual crowding and scrolling text}

Accuracy

All participants in both experiments made some reading errors. Averaged across participants and all experimental trials in Experiment 1, an average of 26.25 errors (SD 20.57) were made by each participant. The average number of errors made per sentence by each participant was 1.25 (SD 2.16). In Experiment 2, an average of 24.31 errors (SD 19.05) were made by each participant. The average number of errors made per sentence by each participant was 0.65 (SD 0.49).

In both experiments, reading accuracy was modulated by spacing condition $\left(\operatorname{Exp} 1 F_{2,22}=14.63, P\right.$ $\left.=.04, \eta_{\mathrm{G}}{ }^{2}=0.16 ; \operatorname{Exp} 2 F_{2,22}=17.95, P<.001, \eta_{\mathrm{G}}{ }^{2}=0.36\right)$. In Experiment 1, participants made an average of 0.72 errors per sentence (SD 0.75) for triple-character spacing, compared with 1.16 errors (SD 0.85) for double-character and 1.83 errors (SD 1.44) for single-character spacing (Figure 3a). Pairwise comparisons showed that single and double inter-word spacing conditions were not significantly different $(P=.06, d=$ 0. 52), but that reading with triple-character inter-word spacing produced significantly fewer errors than both single- $(P=.01, d=0.96)$ and double-character spacing $(P=.01, d=0.54)$. The pattern of results was similar for Experiment 2, where an average of 0.28 errors were made per sentence (SD 0.20) for triplecharacter spacing, compared with 0.69 errors (SD 0.29) for double- and 0.99 errors (SD 0.61) for singlecharacter spacing (Figure 3b). Pairwise comparisons showed that single and double inter-word spacing conditions were not significantly different $(P=.23, d=0.64)$, but that reading with triple-character interword spacing produced significantly fewer errors than both single- $(P<.001, d=1.57)$ and doublecharacter spacing $(P \leq .05, d=1.65)$. In summary, both experiments show that increasing inter-word spacing from one to three characters significantly enhances accuracy for reading eccentrically-viewed scrolling text. 

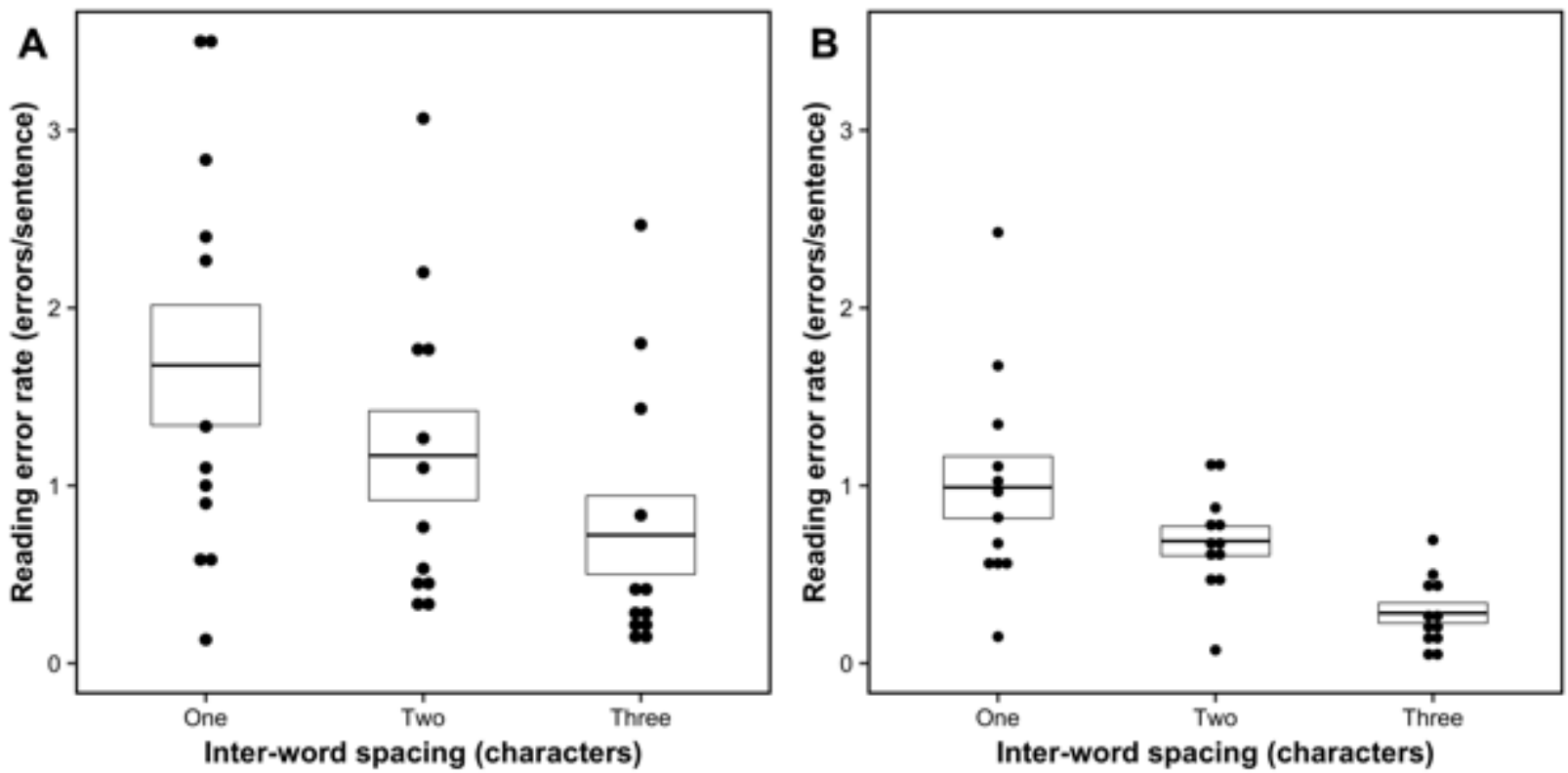

Figure 3. Average number of reading errors made per sentence for inter-word spacing of one, two or three

characters in Experiment 1 (panel a) and Experiment 2 (panel b). Individual dots show individual each participant's performance, and the subdivided box shows group mean and 95\% confidence intervals.

Memory

Memory was defined as the proportion of sentences correctly recalled at the end of each trial.

212 Averaged across all conditions and all participants, the proportion of sentences correctly recalled was

$21373.53 \%$ in Experiment 1 and 80.75\% in Experiment 2. In Experiment 1, 48.33\% (SD 34.86) of sentences

214 were correctly recalled with single-character inter-word spacing. This compares with $80.58 \%$ (SD 24.43)

215 for double-character and 91.67\% (SD 19.46) for triple-character spacing. In Experiment 2, memory scores

216 across spacing conditions were 75.91\% (SD 5.74) for single-character spacing, 80.89\% (SD 5.31) for double-

217 character spacing, and 85.44\% (SD 4.93) for triple-character spacing. As for reading accuracy, there was

218 an effect of spacing condition on recall in both Experiment $1\left(F_{2,22}=13.30, P<.001, \eta_{\mathrm{G}}{ }^{2}=0.34\right)$ and

219 Experiment $2\left(F_{2,22}=11.98, P<.001, \eta_{\mathrm{G}}{ }^{2}=0.37\right)$. For Experiment 1 , pairwise comparisons revealed 220 significantly greater recall for both double- $(P<.01, d=1.07)$ and triple-character $(P<.001, d=1.53)$ inter- 
221 word spacing compared with single-character spacing (Figure 4a). For Experiment 2, pairwise comparisons

222 revealed greater recall for triple-character compared with single-character spacing $(P<.001, d=1.78)$

223 alone (Figure 4b). Note that there were numerical trends in Experiment 2 towards better recall with triple-

224 compared with double-character spacing, and with double- compared with single-character spacing, but

225 these comparisons did not reach statistical significance (double vs. triple $P=.13, d=0.89$; single vs. double

$226 P=.09, d=0.90)$. In summary, both experiments show that, when reading scrolling text, increasing inter-

227 word spacing from one to three characters significantly increases the proportion of sentences correctly 228 recalled.
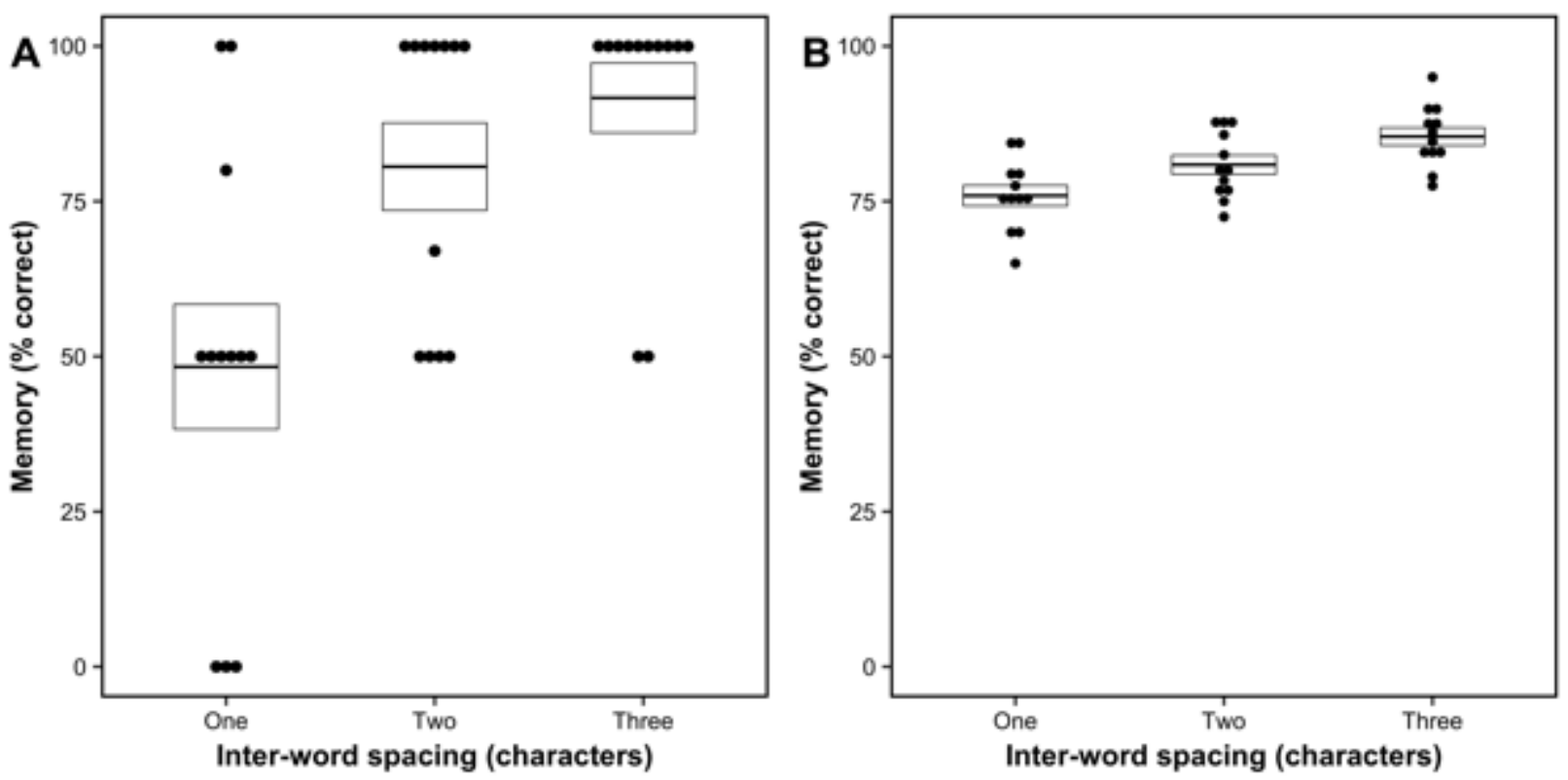

229

230

231

232

233

234

235

236

Figure 4. Percentage of sentences correctly recalled, averaged across all participants for single-, doubleand triple-character inter-word spaces in Experiment 1 (panel a) and Experiment 2 (panel b). Individual dots show each participant's performance, and the subdivided box shows group mean and 95\% confidence intervals. Note that three participants failed to report any sentences correctly in Expt. 1 (single spacing condition). This result is unlikely to reflect a lack of familiarity with the task, as it was the first set of trials for only one of the three non-scoring participants.

\section{Reading Speed}




\section{Visual crowding and scrolling text}

Reading speed is reported here as the number of words read per minute (wpm), where the time

taken to read each sentence was recorded as the temporal interval between screen sentence onset and the final vocalisation of the sentence. Substantial differences between Experiments 1 and 2 were expected for this measure because of the different ways in which text display speeds were set. In Experiment 1, the physical text display speed was matched across the three spacing conditions (to $6.7 \% \mathrm{~s}$ ), resulting in effectively slower presentation speeds in words per minute for more widely spaced text. In Experiment 2, the physical text display speed was adjusted such that the presentation speed in words per minute (approx. $91 \mathrm{wpm}$ ) was the same for each spacing condition (see Methods).

In Experiment 1 reading speed was fastest in the single-character spacing condition (86.63 wpm, SD 7.86), and increasingly slower in the double (76.94, SD 3.56) and triple spacing (71.16, SD 3.12) conditions (see Figure 5a). The decrease in reading speed with increasing inter-word spacing was significant $\left(F_{2,22}=39.03, P<.001, \eta_{\mathrm{G}}{ }^{2}=0.62\right)$. All comparisons between spacing conditions were significant $(P<.05, d>1.5)$.

By contrast, reading speeds in Experiment 2 were, as expected, similar across all three spacing conditions: 84.25 wpm (SD 26.56) with single-character spacing, 86.93 wpm (SD 25.38) with doublecharacter spacing, and 88.33 wpm (SD 21.71) with triple-character spacing (see Figure 5b). There was no significant effect of spacing condition on average reading speed $(P=.74)$. This supports the assertion that improved reading performance with increased inter-word spacing in Experiment 1 cannot be attributed to text display speed alone. 

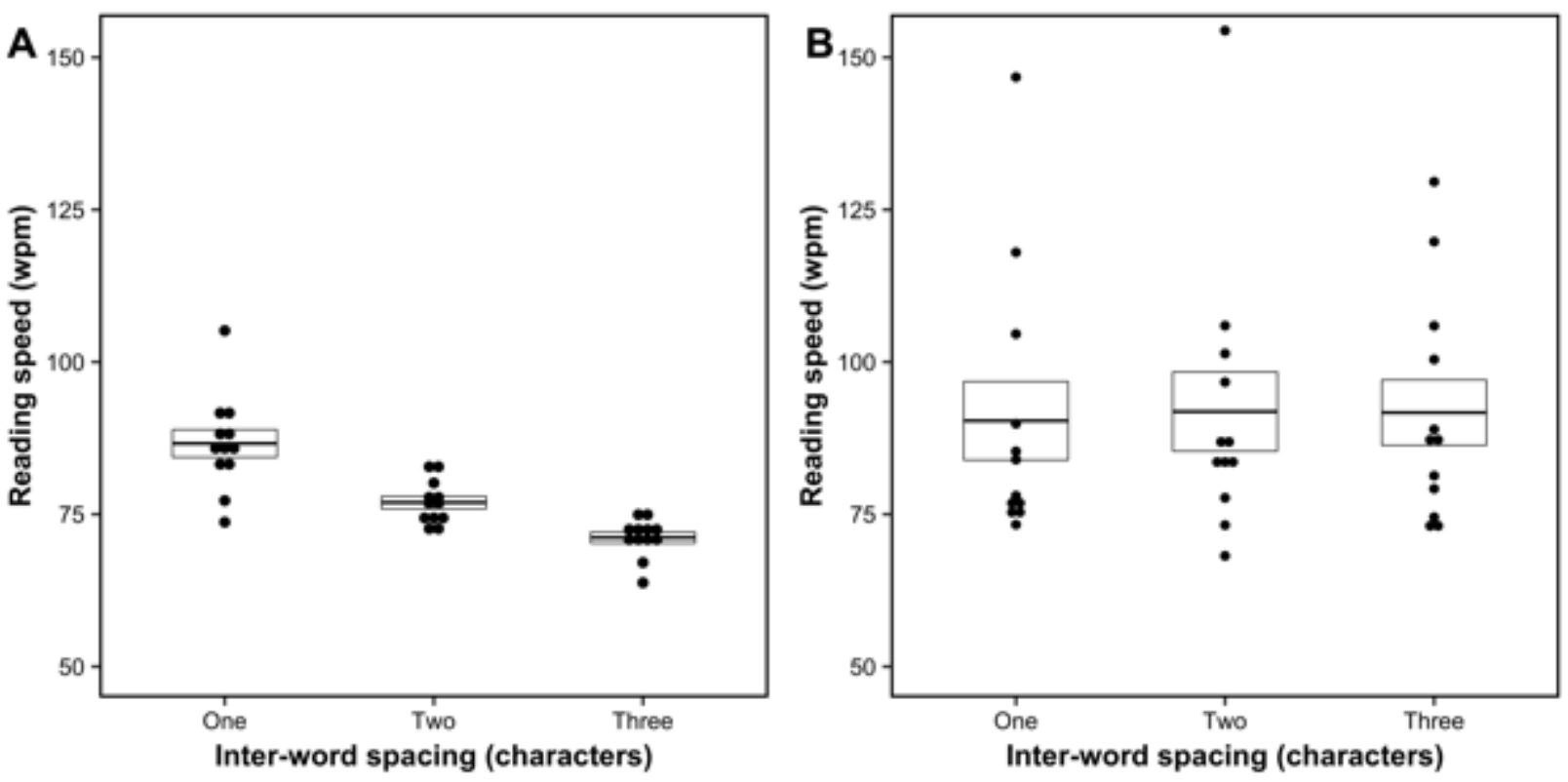

257 Figure 5. Reading speed (words per minute) averaged across all participants for single-, double-and triple-

258 character inter-word spaces in Experiment 1 (panel a) and Experiment 2 (panel b). Individual dots show

259 individual each participant's performance, and the subdivided box shows group mean and 95\% confidence

260 intervals.

261

262

Eye movements

263

A density heat map of fixations, weighted by fixation duration and averaged across all participants,

264 is presented in Figure 6. Data are shown for for single-, double- and triple-character inter-word spaces for

265 Experiment 1 (panel a) and Experiment 2 (panel b). The horizontal broken line, located $4^{\circ}$ above the text,

266 indicates the 'ideal' viewing position for leaving the text unobscured by the artificial scotoma. Note that

267 the distribution of eye fixations remained broadly similar with different word spacings. 

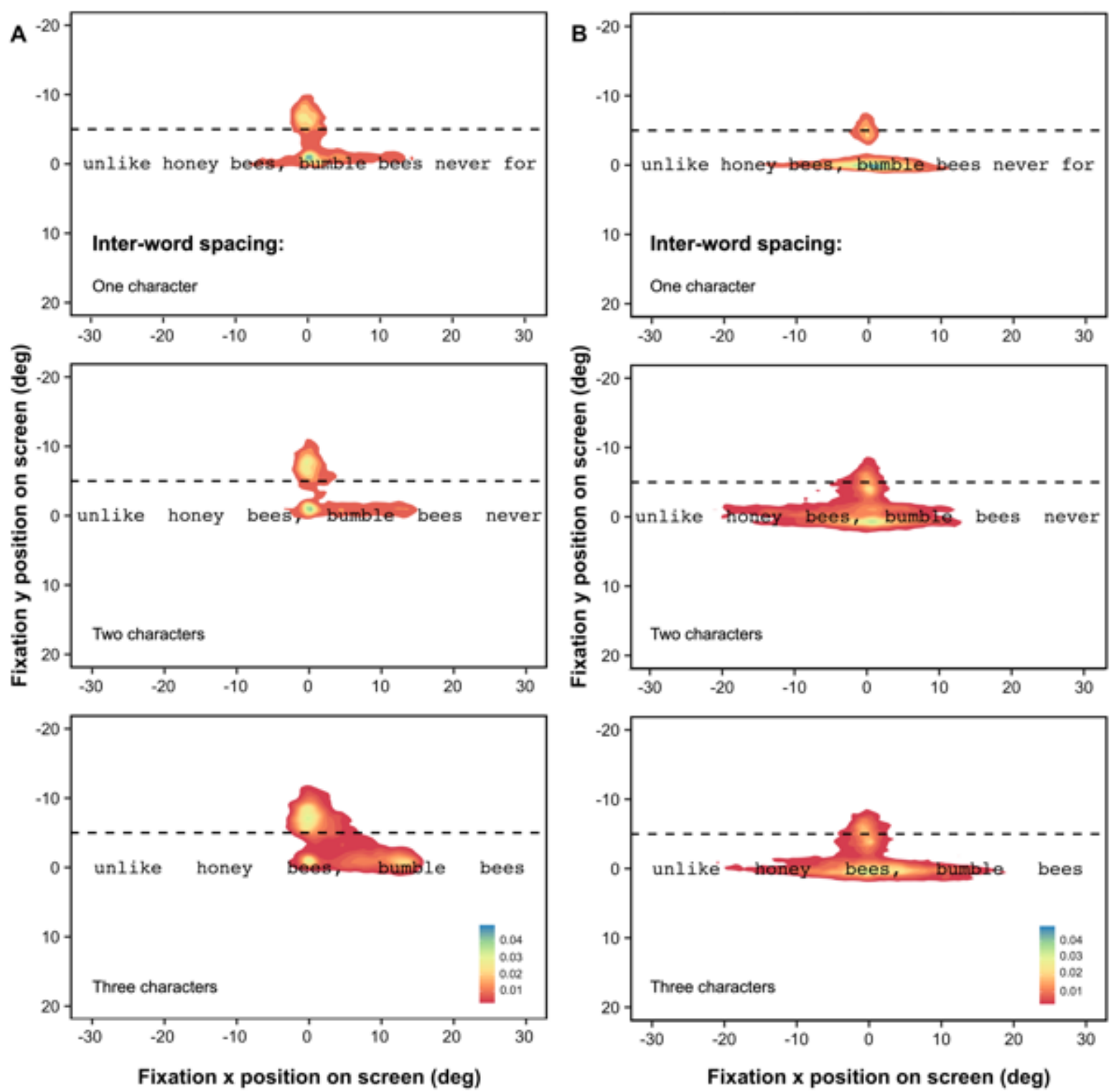

269 Figure 6. Density heat map of fixations, weighted by fixation duration, averaged across all participants for 270 single-, double-and triple-character inter-word spaces in Experiment 1 (panels a) and Experiment 2 (panels

271 b). Densities are calculated using the nonparametric kernel density estimation technique, and brighter

272 colours are associated with higher proportion of fixation time. Screen position is given in degrees of a visual 273 angle, and coordinates $(0,0)$ is the centre of the screen. The horizontal broken line, located $4^{\circ}$ above the 274 text, indicates the 'ideal' viewing position for leaving the text unobscured by the gaze-controlled scotoma. 


\section{Visual crowding and scrolling text}

As can be seen from the heat maps, participants did not maintain the ideal viewing position throughout the experiments - on average, participants spent approximately one third of their viewing time with an ideal fixation location (mean 30\% in both experiments, with a SE of 5\% in Experiment 1 and 4\% in Experiment 2). We note that one participant in Experiment 1 and two participants in Expeirment 2 were able to adhere to the ideal viewing strategy for approximately two-thirds of their viewing time. However, there was no evidence that these few participants achieved any better reading performance. There was also no evidence for any systematic differences in adherence to this viewing strategy across the three spacing conditions.

\section{Discussion}

We investigated the impact of inter-word spacing on performance for reading single lines of horizontally scrolling text in peripheral vision. To ensure that peripheral vision was used for reading, we employed a gaze-contingent central scotoma that covered the entire macular area. We show that reading accuracy (Figure 3) and memory recall (Figure 4) were significantly enhanced with increased inter-word spacing, with the largest improvements observed for triple-character spacing. Our experimental protocol affirmed that these findings were independent of the text presentation speed in words per minute. Given these results, and in general agreement with previous studies, ${ }^{9,18-20}$ we attribute the observed improvements in reading performance with increased inter-word spacing to a reduction in visual crowding $\left(c f^{9}\right)$.

An improvement in reading performance with increased word spacing has been demonstrated in individuals with macular disease, where, for normal contrast static text, double-character inter-word spacing yielded superior reading performance than either single- or triple-character spacing. ${ }^{9}$ In the present study reading performance, in terms of accuracy and memory recall, was better with triplecharacter word spacing than either single- or double-character spacing, a result that may reflect the 
299 increased crowding effect reported with dynamic stimuli. ${ }^{25,34}$ The replication of the improvement across

300 two measures of reading performance and two different experimental protocols demonstrates the 301 reliability of this effect.

It is possible that inter-word separation beyond triple-character spacing may further enhance reading performance. However, given the known trade-off between the beneficial effects of reducing visual crowding and the detrimental consequences of stimuli being shifted into an area of poorer visual acuity, $^{21,29,35}$ it is likely that excessive inter-word spacing (i.e. more than three characters) may be counterproductive, although this remains to be tested. Similarly, although it would also be possible to investigate intra-word (letter) spacing to further reduce visual crowding, evidence from studies with static text suggests that this could disrupt the perception of the word form required for efficient lexical identification. ${ }^{27}$

Dynamic scrolling text necessarily imposes a limit on maximum reading speed as it restricts text availability - words can only be read at the rate at which they appear. With the protocol employed here in Experiment 1, a maximum reading speed of 109 wpm was achievable with single-character word spacing, reducing to $78 \mathrm{wpm}$ for triple-character spacing (see Methods). This reduction may, in part, 314 account for the measured change in reading speed when moving from single- to triple-character inter315 word spacing (see Figure 5a). Nonetheless, although reading speed declined, enhanced word spacing 316 allowed significant improvements in reading accuracy and memory recall. These improvements were 317 replicated in Experiment 2, where using matched display speeds across spacing conditions we confirmed 318 that there was no confound between our reading performance measures and text display speed. This experiment further demonstrated that the observed improvements in reading speed could be maintained at a reasonable reading rate of around 90 words per minute. ${ }^{4,36}$ 
323 suggest that increased inter-word spacing with scrolling text may further improve the overall reading

324 experience of visually compromised individuals. This conclusion may be of particular use to developers of 325 low vision aids and visual rehabilitation practitioners. Some caution may be appropriate in generalising 326 the results here with regard to the retinal area employed for eccentric viewing. In this study we used an $3278^{\circ}$ wide central scotoma, in line with several reading studies of this kind. ${ }^{32,37-47}$ For smaller areas of central 328 vision loss, increased inter-word spacing may be less important as visual crowding is less severe in the 329 region immediately surrounding the fovea. ${ }^{21}$ 


\section{References}

1. Harvey H, Walker R. Reading with Peripheral Vision: A Comparison of Reading Dynamic Scrolling and Static Text with a Simulated Central Scotoma. Vision Res 2014;98:54-60.

2. Walker R. An Ipad App as a Low-Vision Aid for People with Macular Disease. Br J Ophthalmol 2013;97:110-2.

3. Bowers AR, Woods RL, Peli E. Preferred Retinal Locus and Reading Rate with Four Dynamic Text Presentation Formats. Optom Vis Sci 2004;81:205-13.

4. Walker R, Bryan L, Harvey H, et al. The Value of Tablets as Reading Aids for Individuals with Central Visual Field Loss: An Evaluation of Eccentric Reading with Static and Scrolling Text. Ophthalmic Physiol Opt 2016;36:355-512.

5. Harland S, Legge GE, Luebker A. Psychophysics of Reading. XVII. Low-Vision Performance with Four Types of Electronically Magnified Text. Optom Vis Sci 1998;75:183-90.

6. Virgili G, Acosta R, Grover LL, et al. Reading Aids for Adults with Low Vision. Cochrane Database Syst Rev 2013;10:CD003303.

7. Bowers AR, Cheong A, Lovie-Kitchin J. Reading with Optical Magnifiers: Page Navigation Strategies and Difficulties. Optom Vis Sci 2007;84:9-20.

8. Deruaz A, Whatham AR, Mermoud C, Safran AB. Reading with Multiple Preferred Retinal Loci: Implications for Training a More Efficient Reading Strategy. Vision Res 2002;42:2947-57.

9. Blackmore-Wright S, Georgeson MA, Anderson SJ. Enhanced Text Spacing Improves Reading Performance in Individuals with Macular Disease. PLoS ONE 2013;8(11):E80325.

10. Chung STL. Reading Speed Benefits from Increased Vertical Word Spacing in Normal Peripheral Vision. Optom Vis Sci 2004;81:525-35.

11. Pelli DG, Palomares M, Majaj NJ. Crowding Is Unlike Ordinary Masking: Distinguishing Feature 


\section{Visual crowding and scrolling text}

Integration from Detection. J Vis 2004;4:1136-69.

12. Pelli DG, Tillman KA. The Uncrowded Window of Object Recognition. Nat Neurosci 2008;11:1129-35.

13. Brown B. Resolution Thresholds for Moving Targets at the Fovea and in the Peripheral Retina. Vision Res 1972;12:293-304.

14. Crossland MD, Culham LE, Rubin GS. Fixation Stability and Reading Speed in Patients with Newly Developed Macular Disease. Ophthalmic Physiol Opt 2004;24:327-33.

15. Watson G, Berg R. Near Training Techniques. In: Jose RT, Ed. Understanding Low Vision. New York: American Foundation For The Blind; 1983.

16. Paterson KB, Jordan TR. Effects of Increased Letter Spacing on Word Identification and Eye Guidance During Reading. Mem Cognit 2010;38:502-12.

17. Rayner K, Fischer MH, Pollatsek A. Unspaced Text Interferes with Both Word Identification and Eye Movement Control. Vision Res 1998;38:1129-44.

18. Pelli DG, Tillman KA, Freeman J, et al. Crowding and Eccentricity Determine Reading Rate. J Vis 2007;7:1-36.

19. He Y, Legge GE, Yu D. Sensory and Cognitive Influences on the Training-Related Improvement of Reading Speed in Peripheral Vision. J Vis 2013;13:1-14.

20. Latham K, Whitaker D. A Comparison of Word Recognition and Reading Performance in Foveal and Peripheral Vision. Vision Res 1996;36:2665-74.

21. Bouma H. Interaction Effects in Parafoveal Letter Recognition. Nature 1970;226:177-8.

22. Buettner M, Krischer C, Meissen R. Characterization of Gliding Text as a Reading Stimulus. Bull Psychon Soc 1985;23:479-82.

23. Valsecchi M, Gegenfurtner KR, Schütz AC. Saccadic and Smooth-Pursuit Eye Movements during Reading of Drifting Texts. J Vis 2013;13:1-20. 


\section{Visual crowding and scrolling text}

24. Harvey H, Godwin HJ, Fitzsimmons G, et al. Oculomotor and Linguistic Processing Effects in Reading Dynamic Horizontally Scrolling Text. J Exp Psychol Hum Percept Perform 2017;43.

25. Harrison WJ, Remington RW, Mattingley JB. Visual Crowding Is Anisotropic along the Horizontal Meridian during Smooth Pursuit. J Vis 2014;14(1):1-16.

26. Faul F, Erdfelder E, Buchner A, Lang A-G. Statistical Power Analyses Using G*Power 3.1: Tests for Correlation and Regression Analyses. Behav Res Methods 2009;41:1149-60.

27. Chung STL. The Effect of Letter Spacing on Reading Speed in Central and Peripheral Vision. Invest Ophthalmol Vis Sci 2002;43:1270-6.

28. Tarita-Nistor L, Lam D, Brent MH, et al. Courier: A Better Font for Reading with Age-Related Macular Degeneration. Can J Ophthalmol / J Can d'Ophtalmologie 2013;48:56-62.

29. Anstis S. A Chart Demonstrating Variations in Acuity with Retinal Position. Vision Res 1974;14:589-92.

30. Lovie-Kitchin J. Reading with Low Vision: The Impact of Research on Clinical Management. Clin Exp Optom 2011;94:121-32.

31. Legge GE, Ross JA, Luebker A, Lamay JM. Psychophysics Of Reading VIII . The Minnesota LowVision Reading Test. Optom Vis Sci 1989;66:843-53.

32. Aguilar C, Castet E. Gaze-Contingent Simulation of Retinopathy: Some Potential Pitfalls and Remedies. Vision Res 2011;51:997-1012.

33. R Core Team. R: A Language and Environment for Statistical Computing. 2018.

34. Bex PJ, Dakin SC, Simmers AJ. The Shape and Size of Crowding for Moving Targets. Vision Res 2003;43:2895-904.

35. Slattery TJ, Rayner K. Effects of Intraword and Interword Spacing on Eye Movements during Reading: Exploring the Optimal Use of Space in a Line of Text. Atten Percept Psychophys 2013;75:1275-92. 


\section{Visual crowding and scrolling text}

36. Legge GE, Ross JA, Maxwell K, Luebker A. Psychophysics Of Reading. VII. Comprehension in Normal and Low Vision. Clin Vis Sci 1989;4:51-60.

37. Bernard J-B, Scherlen A-C, Castet E. Page Mode Reading with Simulated Scotomas: A Modest Effect of Interline Spacing on Reading Speed. Vision Res 2007;47:3447-59.

38. Scherlen A-C, Bernard J-B, Calabrèse A, Castet E. Page Mode Reading with Simulated Scotomas: Oculo-Motor Patterns. Vision Res 2008;48:1870-8.

39. Varsori M, Perez-Fornos A, Safran AB, Whatham AR. Development of a Viewing Strategy during Adaptation to an Artificial Central Scotoma. Vision Res 2004;44:2691-705.

40. Bertera JH. The Effect of Simulated Scotomas on Visual Search in Normal Subjects. Invest Ophthalmol Vis Sci 1988;29:470-5.

41. Cornelissen FW, Bruin K, Kooijman A. The Influence of Artificial Scotomas on Eye Movements during Visual Search. Optom Vis Sci 2005;82:1-10.

42. Fine EM, Rubin GS. Reading with Central Field Loss: Number of Letters Masked Is More Important than the Size of the Mask in Degrees. Vision Res 1998;39:747-56.

43. Geringswald F, Baumgartner FJ, Pollmann S. A Behavioral Task for the Validation of a GazeContingent Simulated Scotoma. Behav Res Methods 2013;45:1313-21.

44. Kwon M, Nandy AS, Tjan BS. Rapid and Persistent Adaptability Of Human Oculomotor Control in Response To Simulated Central Vision Loss. Curr Biol 2013;23:1663-9.

45. Kwon M, Ramachandra C, Satgunam P, et al. Contour Enhancement Benefits Older Adults with Simulated Central Field Loss. Optom Vis Sci 2012;89:1374-84.

46. Mcllreavy L, Fiser J, Bex PJ. Impact Of Simulated Central Scotomas on Visual Search in Natural Scenes. Optom Vis Sci 2012;89:1385-94.

47. Pidcoe P, Wetzel P. Oculomotor Tracking Strategy in Normal Subjects with and without Simulated Scotoma. Invest Ophthalmol Vis Sci 2006;47:169-78. 